\title{
DEPLETED MAGNETIC FLUX TUBES AS PROBES OF THE IO TORUS PLASMA
}

\author{
C. T. Russell ${ }^{1}$, M. G. Kivelson ${ }^{1}$, W. S. Kurth ${ }^{2}$ and D. A. Gurnett ${ }^{2}$ \\ 'Department of Earth and Space Sciences and Institute of Geophysics and Planetary Physics, \\ University of California Los Angeles, CA 90095-1567, USA \\ ${ }^{2}$ Department of Physics and Astronomy, University of lowa, Iowa City, IA 52242, USA
}

\begin{abstract}
On the initial pass by Io the Galileo spacecraft detected thin tubes of magnetic flux that had stronger fields than their surroundings indicating that they were depleted in their energy content. These tubes have not been seen on every return to the Io torus, only on the passes with the longest stretches of data. They are also not observed much inside the orbit of Io. The statistics of their occurrence gives us further clues as to their origin and constraints on the properties of the Io torus plasmas. The tubes tend to occur in groups separated by hours of elapsed time suggesting a breakup from a larger structure and an episodic event type origin. The tubes also imply that the temperature of the torus is cooler than if all the plasma were due to heavy ion mass loading. The magnetic field excess inside the tubes also decreases with magnetic latitude following the expected latitude dependence of the beta of the plasma.

(C) 2001 COSPAR. Published by Elsevier Science Ltd. All rights reserved.
\end{abstract}

\section{INTRODUCTION}

On December 7, 1995 the Galileo spacecraft passed through the Io torus, recording particles and fields data at full resolution on the tape recorder. Of the many discoveries on this orbit, one was truly unexpected. Several thin flux tubes were found with significantly stronger magnetic fields than their neighbors [Kivelson et al., 1997]. While these tubes certainly contain energetic particles, the torus plasma plausibly is completely missing. Moreover, there is no evidence for erosion of the edges of the tubes such as diffusion would produce. If these tubes are empty of torus plasma, we would expect them to move inward against the general outflow of the torus through their buoyancy. Their rate of occurrence of about $0.4 \%$ indicates that they could replace all of the magnetic flux being carried outward with the torus ions by flowing inward at 300 times the outward velocity. Since the torus plasma need move outward at only a few $\mathrm{m} \mathrm{s}^{-1}$ to maintain a steady state density profile even for mass loading rates of $1000 \mathrm{~kg} \mathrm{~s}^{-1}$ then these tubes need to be moving inward at only a few km s${ }^{-1}$ while their azimuthal velocity should be that of the corotating plasma. Thus their total velocity should be close to that of corotation.

A plausible source for these empty flux tubes is reconnection in the post midnight sector [Russell et al., 1998]. This process produces near vacuum conditions when reconnection proceeds into the lobes and it produces an amount of empty or depleted magnetic flux similar to that loaded up at Io [Russell et al., 2000a]. Thus these empty or depleted flux tubes seem to play an important role in the plasma and magnetic flux circulation in the magnetosphere. A preliminary report on these depleted flux tubes has recently been published [Russell et al., $2000 \mathrm{~b}]$. In this update we examine the statistics of the occurrence of these tubes and their statistical properties using the latest data received from Galileo to learn more about their origin and evolution and to infer some of the properties of the Io torus.

\section{PHYSICS OF DEPLETED FLUX TUBES}

A thin tube of magnetic flux and its plasma content should be in transverse pressure balance with neighboring flux tubes in steady state. Figure 1 shows such pressure balance. The magnitude of the field inside the tube, $B_{b}$, must exceed that in the surrounding plasma, $B_{a}$. If the energy of the thermal plasma is much less than that 




Radial Evolution

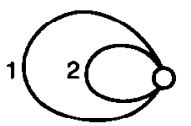

Total flux constant Area of flux tube varies as $\mathrm{A}^{3}$ Radius of flux tube varies as $\mathrm{R}^{3 / 2}$ Corotation velocity varies as $R$ $R_{1}, V_{2} \quad R_{2}, V_{2}$ Duration of $F T$ crossing varies as $R^{1 / 2}$

Fig. 1. Schematic illustration of the physics of a depleted flux tube and its transport.

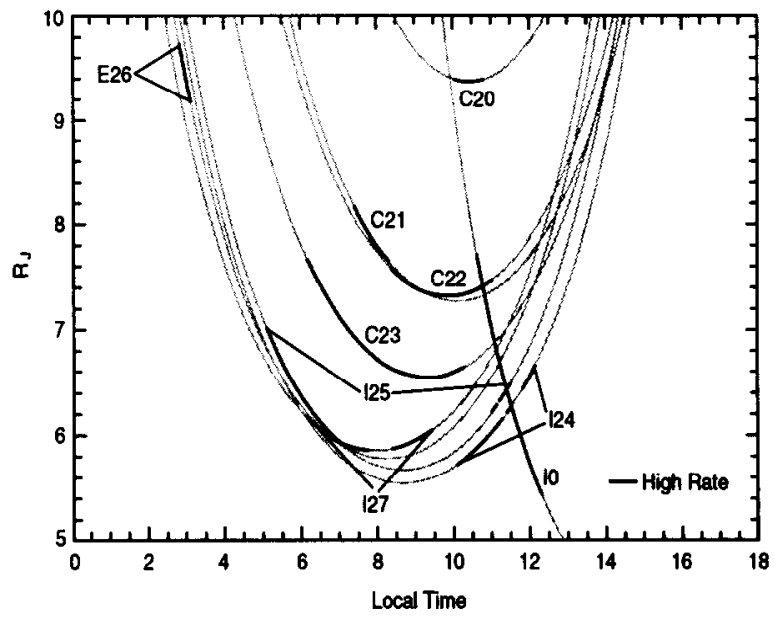

Fig. 2. The radial distances and local times at which the high resolution data required to undertake this study were acquired.

of the magnetic field, then the ratio of the two, the plasma beta is $2 \Delta \mathrm{B} / \mathrm{B}$. If we know the density of the plasma outside the depleted tube we can find the temperature of the plasma from the pressure deficit in the magnetic field.

In a pick-up plasma we expect the plasma pressure to be dominated by the heavy ions because all species receive the same velocity boost. Thus electrons should be cold and protons should be much cooler than sulfur and oxygen ions.

We expect that a flux tube, as it moves inward, will preserve its magnetic flux content. Since the area of the tube varies inversely as the magnetic field strength, the area varies as $R^{3}$ decreases with the inward motion. The radius of the flux tube therefore changes as $R^{3 / 2}$ if the tube remains circular. If the field lines are twisted about the tube axis we expect it to remain circular in cross-section since the magnetic forces are far greater than the plasma forces. The duration of the crossing is presumably due to the corotational velocity since the inward velocity needs to be only a few $\mathrm{km} \mathrm{s}^{-1}$. The corotation velocity varies as $\mathrm{R}$. Thus the duration of the crossing of a flux tube should vary as $R^{1 / 2}$. This variation should not be easily detected given the wide spread seen in the durations and the limited range of radial distances over which measurements are available.

In order to perform this analysis we need high resolution data. Figure 2 shows where such data are available. The longest stretches of high resolution data come from the 10, C20, C22, C23 and 127 passes. Perhaps more than coincidentally it is these passes that provide all our events.

\section{REGION OF OCCURRENCE}

Figure 3 shows examples of depleted flux tubes on 127 where we had 11,580 s of high resolution data principally outside of Io's orbit. The portion inside of Io's orbit was only a few lo radii closer to Jupiter. As we will show below important changes appeared to have occurred in this narrow range of distances, just inside of lo. The top two examples occurred prior to the flyby. Both these examples must have traveled completely around the torus before encountering Io if they are moving inward at the rate we be believe they are traveling. If they moved inward at $1 \mathrm{~km} \mathrm{~s}^{-1}$, and if they were at Io when we encounter them they would be $20 R_{10}$ outside Io's orbit one Jupiter rotation earlier. Thus they probably have not picked up many additional iogenic ions prior to when we see them. In contrast the bottom two cases are observed in the mass-loading region as evidenced by the surrounding ion cyclotron waves. These events are larger than usual because of surrounding enhanced torus plasma, but the earlier two events are equally smaller than normal. We discuss this phenomenon in the next section. Here we wish to concentrate on where we see the depleted flux tubes and on their occurrence rates. Table 1 shows the number of seconds of coverage on each of our passes divided into two radial ranges, outside and inside Io's orbit. There is significant coverage inside lo's orbit only on the J0 and I24 passes. No depleted flux tubes were seen in those over $11,500 \mathrm{~s}$. Outside Io's orbit, events were seen on passes with $24,720,17,220,11,580,9,540$ and $7,260 \mathrm{~s}$ but not on passes with 7070,6720 and 3639 s. On no orbit with detection was only one event detected. On orbits with detection there were 2 to 10 events. 
The statistics in this table can be best explained by a model in which depleted flux tubes appear in groups that are created every few hours and drift inward as a bunch. If the duration of the observation period is shorter than about 2 hours it is improbable to run into a flock of these flux tubes but for periods of three or more hours it is most probable to encounter them. We envision these groupings originating in a single event producing a large tube followed by a breakup of the large tube into smaller ones. The implied time scale is similar to that produced in jovian substorms.

Lastly, we note the grand average occurrence rate, given in Table 1 , of $0.37 \%$. This rate implies that the inward motion of the flux tubes is about 300 times the outward flux transport of the neighboring torus flux tubes if these flux tubes are providing the return flux from the substorm reconnection region.

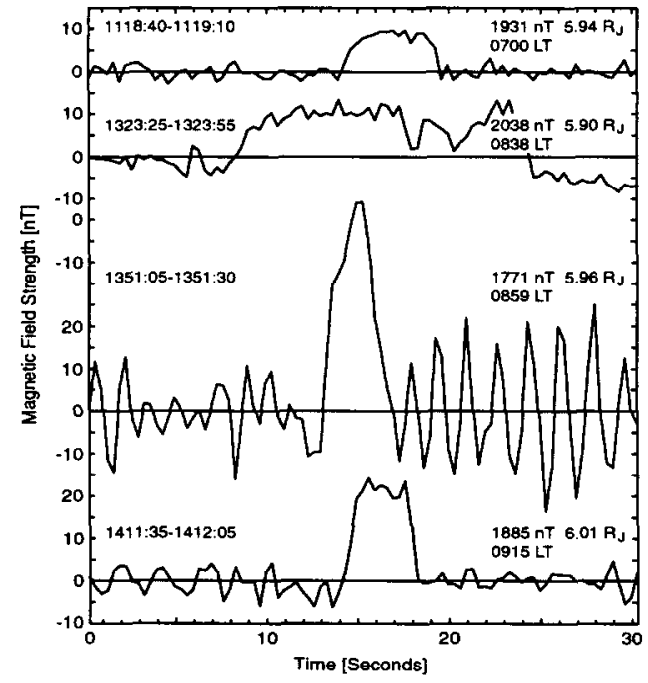

Fig. 3. The magnetic field strength versus time in four sample depleted flux tubes from pass I27.

Table 1. Occurrence rates of depleted flux tubes

\begin{tabular}{rrrrrrrrr}
\hline & \multicolumn{2}{c}{ Coverage [s] } & \multicolumn{2}{c}{ Depleted Flux Tube [s] } & \multicolumn{2}{c}{$\%$ Depleted FT } & Events \\
Pass & $>5.9 \mathrm{R}_{\mathrm{J}}$ & $<5.9 \mathrm{R}_{\mathrm{J}}$ & $>5.9 \mathrm{R}_{\mathrm{J}}$ & $<5.9 \mathrm{R}_{\mathrm{J}}$ & $>5.9 \mathrm{R}_{\mathrm{J}}$ & $<5.9 \mathrm{R}_{\mathrm{J}}$ & \\
\hline J0 & 9540 & 9420 & 29 & 0 & $0.30 \%$ & $0.00 \%$ & 10 \\
C20 & 7260 & 0 & 8 & - & $0.11 \%$ & - & 2 \\
C21 & 6720 & 0 & 0 & - & $0.00 \%$ & - & 0 \\
C22 & 17220 & 0 & 130 & - & $0.75 \%$ & - & 2 \\
C23 & 24720 & 0 & 138 & - & $0.56 \%$ & - & 4 \\
I24 & 4690 & 2140 & 0 & 0 & $0.00 \%$ & $0.00 \%$ & 0 \\
I25 & 7070 & 0 & 0 & - & $0.00 \%$ & - & 0 \\
E26 & 3639 & 0 & 0 & - & $0.00 \%$ & - & 0 \\
I27 & 11580 & 0 & 41 & - & $0.35 \%$ & - & 9 \\
\hline Total & 92439 & 11560 & 346 & 0 & $0.37 \%$ & $0.00 \%$ & 27 \\
\hline
\end{tabular}

\section{FLUX TUBE DISAPPEARANCE}

Table 2 lists the properties of sample depleted flux tubes from the passes discussed in the previous section. The beta of the surrounding flux tubes relative to that in the depleted flux tube is shown in the middle column. These range from 0.007 to 0.029 . Figure 4 shows the radial location of these flux tubes. The "high" beta events occur just outside of Io's orbit and the low beta events tend to occur inside Io's orbit. If in fact the tubes are moving radially inward as we suppose, then the rapid disappearance of the magnetic field enhancement when the tubes move in past Io is due to the fact that the ambient magnetic flux inside of Io's orbit contains very little warm plasma pressure i.e. it is effectively depleted as well and there is no contrast. Thus the inward moving tubes simply fade into the background with no alteration. If they drift through the mass loading region they receive a dose of hot ions but it may require several such passes through the mass loading region in order for the depleted flux tubes to reach the energy density of the torus plasma.

\section{ION TEMPERATURES}

In Table 2 we list the ion temperature calculated from the change in pressure from inside to outside the flux tube and the electron density outside the tube. We assume that highly energetic particles can drift into the flux tubes and are the same inside and outside the tubes. The calculation refers only to the torus ions, which appear not to enter the tubes or else the tubes would have rounded edges. There is a vast range of deduced temperature from 27 to $203 \mathrm{eV}$. 


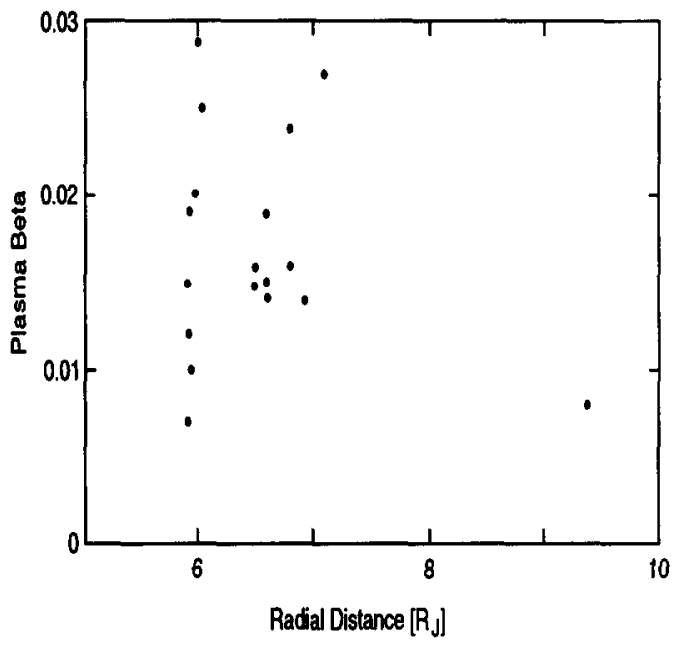

Fig. 4. The inferred plasma beta versus radial distance.

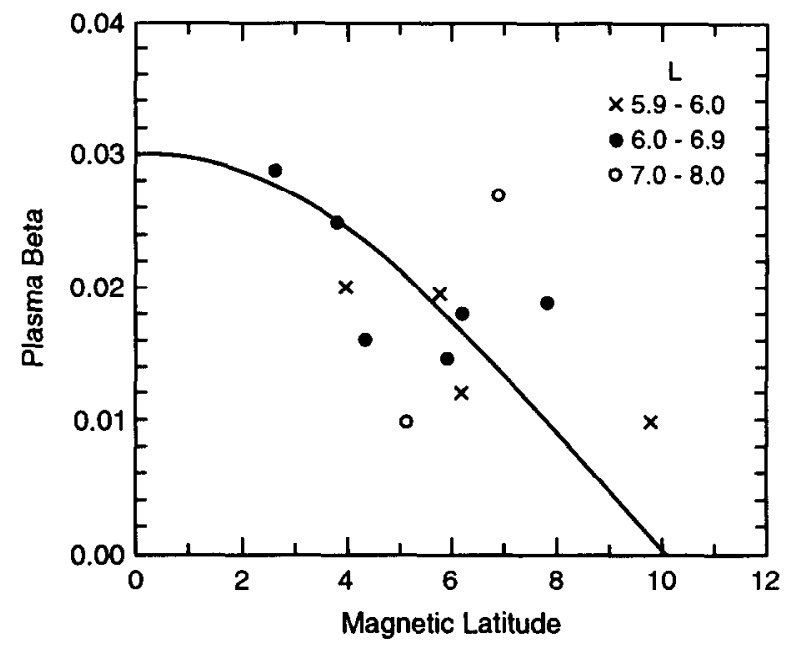

Fig. 5. Inferred plasma beta versus magnetic latitude.

However none of these perpendicular temperatures approaches that of a $\mathrm{S}^{+}$ion picked up at $100 \mathrm{~km} / \mathrm{s}$, the fastest velocities seen near Io, or the velocity of an ion picked up well outside of Io's orbit $\left(9 R_{J}\right)$. This value is $1670 \mathrm{eV}$. To reduce the average temperature to $200 \mathrm{eV}$ one would have to assume that the typical mass of a picked up ion was $16 \mathrm{amu}$ and the velocity of pickup was $50 \mathrm{~km} / \mathrm{s}$. However, even $200 \mathrm{eV}$ is an extreme value in our distribution and $50 \mathrm{~km} / \mathrm{sec}$ is on the low side of average velocity of the torus plasma relative to Io and it would be difficult to produce much $\mathrm{O}$ without a similar amount of $\mathrm{S}$ from the $\mathrm{SO}$ and $\mathrm{SO}_{2}$ around Io. Thus there may be a significant source of hydrogen as well, perhaps from $\mathrm{H}_{2} \mathrm{~S}$ [Russell and Kivelson, 2000], but observations by Galileo seem to limit the production rate of protons about $10 \%$ of that of $\mathrm{O}^{+}$[Frank and Patterson, 1999].

The inferred temperature as a function of radial distance is plotted in Figure 4 of Russell and Kivelson [2000] and compared with direct measurements by Galileo and Voyager. The temperatures fall in the range reported by Voyager but there is much variation and the temperatures are much less than expected for heavy ion pickup. On the J0 pass the temperatures found by the calculation based on the plasma beta and density does agree with the ion temperature reported by Crary et al. [1998]. A possible answer for the orbit to orbit changes is a variable source of protons in the pickup. Another possibility is a variable velocity of the plasma in the altitude range where the mass loading first occurs and fast neutrals produced. This region could be low in the exosphere where charge exchange can occur readily [Russell et al., 2001]. We note that well away from Io in the radial direction one would expect that Coulomb collisions with electrons followed by UV radiation would cool the ions but this mechanism is less able to predict the cool ions right at $5.9 \mathrm{R}_{\mathrm{J}}$.

The torus plasma is produced by Io and stays mainly in the range of magnetic latitudes that lo occupies as Jupiter spins. Thus the torus is densest at the magnetic equator and becomes quite rarefied about $10^{\circ}$ latitude. Our determination of beta in Table 2 involves only the change in magnetic field across the rope boundary and the total field strength. Thus if our hypothesis on the nature of the depleted flux tubes is correct, then the beta values should drop to zero at about $10^{\circ}$ latitude. Figure 5 shows the $\beta$ values of Table 2 plotted versus latitude together with the function $0.3 \cos (9 \lambda)$ where $\lambda$ is the magnetic latitude in degrees. While there is some scatter, it is clear that the deduced $\beta$ values vary with latitude as we would expect.

\section{CONCLUSIONS}

The Galileo magnetometer has found isolated flux tubes with enhanced magnetic field strengths in the inner magnetosphere of Jupiter but not closer to Jupiter than Io's orbit. These flux tubes appear to be depleted of their torus plasma. Using the difference in magnetic energy between these tubes and their neighbors we infer that the plasma beta near lo is about 0.02 but it falls rapidly inside Io's orbit. Outside Io's orbit it is greatest near the magnetic equator and decreases with magnetic latitude so that it approaches zero near $10^{\circ}$ latitude. Using the electron density measurements from the plasma wave spectrometer we translate these betas into ion temperatures of 30-100 eV consistent with Voyager but these temperatures are lower than expected for heavy ion pickup and they are quite variable. Possible explanations include a variable source of protons at Io and changes in the plasma velo- 
Table 2. Observed and inferred properties of sample depleted flux tubes

\begin{tabular}{cccccccccc}
\hline Time & & $\begin{array}{c}\mathrm{B} \\
{[\mathrm{nT}]}\end{array}$ & \multicolumn{1}{c}{$\begin{array}{c}\Delta \mathrm{B} \\
{[\mathrm{nT}]}\end{array}$} & $\begin{array}{c}\Delta \mathrm{E} \\
{\left[10^{10} \mathrm{eVm}^{-3}\right]}\end{array}$ & $\beta$ & $\begin{array}{c}\text { Density } \\
{\left[10^{8} \mathrm{~m}^{-3}\right]}\end{array}$ & $\begin{array}{c}\mathrm{T} \perp \\
{[\mathrm{eV}]}\end{array}$ & $\begin{array}{c}\mathrm{R} \\
{\left[\mathrm{R}_{\mathrm{J}}\right]}\end{array}$ & $\begin{array}{c}\text { Mag. } \\
\text { Lat. }\end{array}$ \\
\hline \multirow{2}{*}{$12 / 7 / 95$} & 1734 & 1695 & 24.3 & 20.5 & 0.029 & 38 & 54 & 6.0 & $3.2^{\circ}$ \\
& 1712 & 1490 & 11.0 & 8.2 & 0.016 & 32 & 27 & 6.3 & $4.4^{\circ}$ \\
& 1646 & 1300 & 9.4 & 6.1 & 0.015 & 23 & 27 & 6.6 & $5.9^{\circ}$ \\
& 1631 & 1200 & 10.8 & 6.4 & 0.018 & 18 & 36 & 6.8 & $6.2^{\circ}$ \\
$5 / 03 / 99$ & 1610 & 1080 & 14.4 & 7.7 & 0.027 & 11 & 68 & 7.1 & $6.9^{\circ}$ \\
& 1756 & 440 & 1.8 & 3.9 & 0.008 & 1.1 & 36 & 9.4 & $-1.2^{\circ}$ \\
$8 / 12 / 99$ & 1654 & 453 & 4.5 & 1.0 & 0.020 & 0.5 & 203 & 9.4 & $-5.9^{\circ}$ \\
$9 / 14 / 99$ & 1150 & 920 & 4.5 & 2.1 & 0.010 & 2.0 & 110 & 7.3 & $-5.1^{\circ}$ \\
$2 / 22 / 00$ & 114 & 1375 & 13.0 & 8.9 & 0.019 & 11 & 81 & 6.6 & $7.9^{\circ}$ \\
& 119 & 1925 & 9.5 & 9.1 & 0.010 & 13 & 68 & 5.94 & $-9.8^{\circ}$ \\
& 1307 & 2042 & 7.0 & 7.1 & 0.007 & 12 & 61 & 5.88 & $-7.0^{\circ}$ \\
& 1322 & 2034 & 15.5 & 15.7 & 0.015 & 13 & 118 & 5.89 & $-6.2^{\circ}$ \\
& 1323 & 2035 & 12.5 & 12.6 & 0.012 & 14 & 90 & 5.90 & $-6.2^{\circ}$ \\
& 1329 & 2036 & 19.0 & 19.2 & 0.019 & 14 & 137 & 5.91 & $-5.8^{\circ}$ \\
& 1402 & 1896 & 19.0 & 17.9 & 0.020 & 15 & 117 & 5.98 & $-4.0^{\circ}$ \\
& 1412 & 1830 & 23.0 & 20.9 & 0.025 & 15 & 137 & 6.06 & $-3.9^{\circ}$ \\
\hline
\end{tabular}

city in the region where the ions are first accelerated. Outside the immediate massloading region collisional cooling and UV radiation are also responsible for the low temperature. Finally we note that the depleted flux tubes appear in groups separated in time by about 3 hours consistent with formation by jovian substorms and the subsequent inward motion of the flux and breakup into narrow flux tubes. These flux tubes therefore plausibly return the emptied flux tubes from the tail region to the inner magnetosphere to undergo further mass loading and repeat the mass transport cycle.

\section{ACKNOWLEDGMENTS}

This research was supported by the National Aeronautics and Space Administration under grant JPL 958510 administered by the Jet Propulsion Laboratory and NAG5-8064.

\section{REFERENCES}

Crary, F. J., F. Bagenal, L. A. Frank and W. R. Paterson, Galileo plasma spectrometer measurements of composition and temperature in the Io plasma torus, J. Geophys. Res., 103, 29,359-29,370, 1998.

Frank, L. A., and W. R. Paterson, Production of hydrogen ions at Io, J. Geophys. Res., 104, 10,345-10,354, 1999.

Kivelson, M. G., K. K. Khurana, C. T. Russell and R. J. Walker, Intermittent short-duration magnetic field anomalies in the Io torus, Evidence for plasma interchange, Geophys. Res. Lett. 24, 2127-2130, 1997.

Russell, C. T., K. K. Khurana, D. E. Huddleston and M. G. Kivelson, Localized reconnection in the near Jovian magnetotail, Science, 280, 1061-1064, 1998.

Russell C. T., M. G. Kivelson, K. K. Khurana and D. E. Huddleston, Circulation and dynamics in the jovian magnetosphere, Adv. Space Res., 26(10), 1671-1676, 2000a.

Russell, C. T., M. G. Kivelson, W. S. Kurth and D. A. Gurnett, Implications of depleted flux tubes in the jovian magnetosphere, Geophys. Res. Lett., 27, 3133-3136, $2000 \mathrm{~b}$.

Russell, C. T., K. K. Khurana, M. G. Kivelson and D. E. Huddleston, Substorms at Jupiter: Galileo observations of transient reconnection in the near tail, Adv. Space Res., 26(10), 1499-1504, 2000.

Russell, C. T., X. Blanco-Cano, R. J. Strangeway, Y. L. Wang and J. Raeder, A mechanism for the production of a disk-shaped neutral source cloud at Io, Adv. Space Res., submitted, 2001. 